\title{
Characteristics of Transplant Recipients Who Developed Influenza in 2007-08 Despite Influenza Vaccination
}

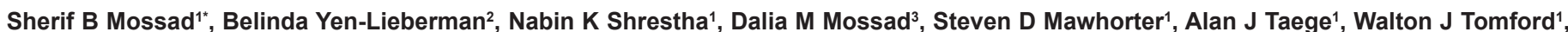
Lucileia T Johnson ${ }^{1}$, Michelle Lard ${ }^{1}$, Thomas G Fraser ${ }^{1}$, Sorabh Dhar ${ }^{1}$, David Van Duin ${ }^{1}$, Rabin K Shrestha ${ }^{1}$, Susan J Rehm ${ }^{1}$, Robin K Avery ${ }^{1}$ and Steven M Gordon'1

${ }^{1}$ Department of Hypertension and Nephrology, Glickman Urological and Kidney Institute, Cleveland Clinic, USA

${ }^{2}$ Division of Infectious Diseases, Detroit Medical Center, Wayne State University, USA

${ }^{3}$ Division of Infectious Disease (Transplant/Oncology), Johns Hopkins, USA

\section{Abstract}

Background: Influenza vaccination may be less immunogenic in transplant recipients than in healthy people. Influenza $A$ (H1N1) and B circulating viruses during the 2007-2008 epidemic were different from those contained in that season's vaccine. In that epidemic, influenza vaccine effectiveness against culture-confirmed influenza was $44 \%$.

Objective: To describe clinical, immunological, and virological characteristics of 18 transplant recipients who developed influenza during the 2007-08 epidemic despite influenza vaccination (tx-vac-flu), and compare them to 6 transplant recipients who developed influenza in the absence of influenza vaccination (tx-no vac-flu), 12 previously healthy people who developed influenza (healthy-flu), and 13 transplant recipients who received influenza vaccination and did not develop influenza (tx-vac-no flu).

Methods: Case ascertainment was through microbiology and electronic medical records. A case of influenza was defined by a clinical presentation of an influenza-like illness, plus a positive influenza A or B multiplex real time polymerase chain reaction (Prodesse, Inc. Waukesha, WI) on a nasopharyngeal swab.

Results: Of the 36 patients with influenza, 22 had influenza A, and 15 had influenza B (1 transplant recipient had both serotypes simultaneously). Types of transplant were lung (11), hematopoietic stem cell (8), heart (7), liver (3), kidney (3), kidney + pancreas (3), liver + kidney (1), and liver + pancreas (1). Patients in the tx-vac-flu group were significantly older than patients in the tx-no vac-flu group [median 61 vs. 50.5 year, $(P=0.02)$, the healthy-flu group [median 49.5 years $(P=0.04)$ ], and the tx-vac-no flu group [median 53 years $(P=0.02)$ ]. Influenza occurred 1,410 $(261-3,467)$ days \{median [interquartile $(I Q R)$ range]\} after transplant in the tx-vac-flu group, compared to $175(40-1,064]$ days in the tx-no vac-flu group $(P=0.18)$. Influenza occurred 114 days (median [IQR 99-137]) after vaccination in the tx-vac-flu group. Immunoglobulin $\mathrm{G}$ levels and immune function assay levels were not significantly different between the 3 transplant groups. There were no statistically significant differences in the incidence of fever, headache, cough, rhinorrhea, sore throat, malaise, shortness of breath, exposure to contacts with similar symptoms, presence of infiltrates on chest roentgenograms, or the estimated influenza viral loads among the 3 groups who had influenza. Patients in the tx-vac-flu group were treated with oseltamivir significantly more frequently than the healthy-flu group $[94 \%$ vs. 50\% $(P=0.0006)]$, but not the tx-no vac-flu group [100\% $(P=0.7)]$. Duration of treatment with oseltamivir was not significantly different among the 3 groups who had influenza. Patients in the tx-vac-flu group had concomitant infections significantly more frequently than the healthy-flu group [44\% vs. 8\% ( $P=0.043)$, but not the tx-no vac-flu group. Patients in the tx-vac-flu group developed pneumonia, and were hospitalized for management of influenza significantly more frequently than patients in the healthy-flu group $(\mathrm{P}=0.031$ and $\mathrm{P}=0.00007$; respectively). Only one patient $(6 \%)$ in the tx-vac-flu group and none in the tx-no vac-flu or healthy-flu groups required admission to an intensive care unit and mechanical ventilation following influenza. No patients died as a result of influenza.

Conclusions: Influenza vaccination did not alter clinical presentation of influenza in transplant recipients, but these patients were hospitalized and developed pneumonia more frequently than healthy people. Transplant recipients who developed influenza despite influenza vaccination were not more immunosuppressed than transplant recipients who were vaccinated and did not develop influenza. Transplant recipients who developed influenza despite influenza vaccination were more likely to have concomitant infections than healthy people with influenza.

Keywords: Influenza; Vaccination; Transplantation

\section{Introduction}

Influenza virus infection may be associated with severe illness \& significant complications in solid organ transplant (SOT) and hematopoietic stem cell transplant (HSCT) recipients [1,2]. Although influenza vaccination remains the primary preventive measure, vaccination may be less immunogenic in transplant recipients than healthy people, as assessed by either the humoral or cell-mediated immune responses $[3,4]$. Nevertheless, several studies conducted in SOT and HSCT recipients [5-9] have shown some degree of protection from influenza and its complications by vaccination. National guidelines recommend influenza vaccination for SOT and HSCT recipients $[10,11]$

Several factors impact vaccine responsiveness in this population; probably most importantly is the net state of immunosuppression; rather than particular immunosuppressive agents [12]. The prophylactic and therapeutic efficacy of antiviral agents in SOT and HSCT recipients with influenza has been shown in case series [13-15].
In addition, a randomized, double-blind, placebo-controlled, multicenter study of seasonal prophylaxis with oseltamivir in SOT recipients showed a protective efficacy of $57 \%$ [16]. A treatment study comparing conventional and high dose oseltamivir in immunocompromised patients; including SOT and HSCT recipients is underway [17],

*Corresponding author: Sherif Beniameen Mossad, Department of Hypertension and Nephrology, Glickman Urological and Kidney Institute, Cleveland 9500, Euclid Avenue. G-21, Cleveland, OH 44195, USA, Tel: 216-445-2572 Fax: 216-445-9446; E-mail: mossads@ccf.org

Received March 22, 2013; Accepted March 29, 2013; Published March 31, 2013 Citation: Mossad SB, Yen-Lieberman B, Shrestha NK, Mossad DM, Mawhorter SD, et al. (2013) Characteristics of Transplant Recipients Who Developed Influenza in 2007-08 Despite Influenza Vaccination. J Vaccines Vaccin 4: 177 doi:10.4172/2157-7560.1000177

Copyright: (c) 2013 Mossad SB, et al. This is an open-access article distributed under the terms of the Creative Commons Attribution License, which permits unrestricted use, distribution, and reproduction in any medium, provided the original author and source are credited. 
however changing patterns of antiviral resistance must be taken into account in terms of applicability to future influenza seasons. Treatment of influenza in SOT and HSCT recipients occasionally fails even if the viral strain is sensitive to the antiviral drug used [18]; due to underlying immunosuppression or altered bioavailability. Severe lymphopenia is associated with higher mortality in HSCT recipients with influenza pneumonia [2]. The 2009 influenza A/H1N1 influenza pandemic illustrated the significant morbidity, despite antiviral therapy, seen in SOT $[19,20]$ and HSCT [21] recipients; serving as "sentinel chickens" [22]

Seasonal influenza A/H1N1 and B circulating viruses during the 2007-2008 epidemics were different from the serotypes contained in that season's vaccine [23]. Despite the suboptimal match between two of the three vaccine strains and the circulating influenza strains, overall vaccine effectiveness against culture-confirmed influenza was $44 \%$, with higher estimates (54\%) among healthy persons aged 5-49 years.

The current study was conducted to describe the clinical and immunological characteristics of 18 transplant recipients who developed influenza during the 2007-08 epidemic despite influenza vaccination (tx-vac-flu), and compare them to 6 transplant recipients who developed influenza in the absence of influenza vaccination (tx-no vac-flu), 12 previously healthy people who developed influenza (healthyflu), and 13 transplant recipients who received influenza vaccination and did not develop influenza (tx-vac-no flu). To our knowledge, there were no other tx-vac-flu patients in our transplant program during the 2007-2008 epidemics. Patients in the 3 other groups were randomly identified by the authors. The 4 groups were selected based on the above characteristics; they were not matched.

\section{Methods}

After Institutional Review Board approval, cases of influenza were retrospectively ascertained through the microbiology laboratory records, and clinical information was collected from the electronic medical records (EMR). A case of influenza was defined by a clinical presentation of an influenza-like illness (ILI) consisting mainly of fever and cough, but including other symptoms, such as headache and rhinorrhea, plus a positive influenza A or B reverse-transcriptase polymerase chain reaction (RT-PCR) on a nasopharyngeal swab. Specimens were collected using sterile plastic application swabs and placed in $3 \mathrm{~mL}$ MicroTest ${ }^{\mathrm{m}} \mathrm{M} 4^{\circ}$ media (Remel; KS, USA). The onestep multiplex RT-PCR ProFlu-plus assay (Prodesse; WI, USA) was performed according to a previously published protocol [24].

Estimates of influenza viral loads were inferred from cycle threshold (CT) for RT-PCR. Cycle threshold is the number of PCR cycles needed to turn positive for influenza A or B in each sample. The lower the CT number, the higher the viral load. Prespecified outcomes of interest included the development of pneumonia, hospitalization, admission to the intensive care unit, need for mechanical ventilation, and death.

The tx-vac-flu group was always the comparator group. A two-tailed $\mathrm{t}$-test was used for comparing means and range of continuous variables. A two-tailed Mann-Whitney U Test was used for comparing medians and interquartile range $[\mathrm{IQR}]$ of continuous variables when the data where skewed. A two-tailed Mid-P exact test was used for comparing categorical variables. P-value $<0.05$ was considered significant.

\section{Results}

\section{Demographic data}

Lung transplant recipients comprised the biggest proportion of transplant patients (11), followed by HSCT (8), heart (7), liver (3), kidney (3), kidney + pancreas (3), liver + pancreas (1), and liver + kidney (1) recipients. In 2008, 168 kidney, 152 hematopoietic stem cell, 147 liver, 60 heart, 57 lung, 31 pancreas, and 4 intestinal transplants were done at our institution. Patients in the tx-vac-flu group were significantly older than patients in the tx-no vac-flu group $(\mathrm{P}=0.02)$, the healthy-flu group $(\mathrm{P}=0.04)$, and the tx-vac-no flu group $(\mathrm{P}=0.02)$. There were no significant differences in gender. There was no significant difference between the tx-vac-flu group and the tx-no vac-flu group in the interval between transplantation and influenza $(\mathrm{P}=0.18)$. Median duration between influenza vaccination and development of influenza in the tx-vac-flu group was 114 [IQR 99-137] days. One patient, a nurse, in the healthy-flu group had received influenza vaccination earlier in

\begin{tabular}{|c|c|c|c|c|}
\hline & \multicolumn{4}{|c|}{ Group } \\
\hline & $\begin{array}{l}\text { Tx-vac-flu } \\
(n=18)\end{array}$ & $\begin{array}{l}\text { Tx-no vac-flu } \\
\quad(n=6)\end{array}$ & $\begin{array}{l}\text { Healthy-flu } \\
\quad(n=12)\end{array}$ & $\begin{array}{l}\text { Tx-vac-no flu } \\
(n=13)\end{array}$ \\
\hline $\begin{array}{c}\text { Type of Transplant } \\
\text { Liver } \\
\text { Heart } \\
\text { Kidney } \\
\text { Lung } \\
\text { HSCT } \\
\text { Kidney + pancreas } \\
\text { Liver + pancreas } \\
\text { Liver + kidney }\end{array}$ & $\begin{array}{l}1 \\
5 \\
2 \\
7 \\
2 \\
1 \\
0 \\
0\end{array}$ & $\begin{array}{l}2 \\
1 \\
0 \\
0 \\
3 \\
0 \\
0 \\
0\end{array}$ & $N / A$ & $\begin{array}{l}0 \\
1 \\
1 \\
4 \\
3 \\
2 \\
1 \\
1\end{array}$ \\
\hline Age (years) \{median [IQR]\} & $61[54-65]$ & $50.5[44-57]^{*}$ & $49.5[34-60]^{\dagger}$ & $53[47-59]^{\#}$ \\
\hline $\begin{array}{c}\text { Gender } \\
\text { Female } \\
\text { Male }\end{array}$ & $\begin{array}{c}7 \\
11\end{array}$ & $\begin{array}{l}1 \\
5\end{array}$ & $\begin{array}{l}7 \\
5\end{array}$ & $\begin{array}{l}4 \\
9\end{array}$ \\
\hline $\begin{array}{c}\text { Duration between transplant } \\
\text { and influenza (days) }\{\text { median }[I Q R]\}\end{array}$ & $1,410[261-3,467]$ & $175[40-1,064]^{* *}$ & $\mathrm{~N} / \mathrm{A}$ & $\mathrm{N} / \mathrm{A}$ \\
\hline
\end{tabular}

Tx-vac-flu=Transplant recipients who developed influenza despite influenza vaccination

Tx-no vac-flu=Transplant recipients who developed influenza in the absence of influenza vaccination

Healthy-flu=Healthy people who developed influenza

Tx-vac-no flu=Transplant recipients who received influenza vaccination and did not develop influenza

$\mathrm{HSCT}=$ Hematopoietic stem cell transplant. IQR=Interquartile range. N/A=not applicable

${ }^{*} \mathrm{P}=0.02,{ }^{+} \mathrm{P}=0.04,{ }^{*} \mathrm{P}=0.02,{ }^{*} \mathrm{P}=0.18$; all compared to the tx-vac-flu group.

Table 1: Demographic data. 
Citation: Mossad SB, Yen-Lieberman B, Shrestha NK, Mossad DM, Mawhorter SD, et al. (2013) Characteristics of Transplant Recipients Who Developed Influenza in 2007-08 Despite Influenza Vaccination. J Vaccines Vaccin 4: 177. doi:10.4172/2157-7560.1000177

the season. However; none of the other patients in the healthy-flu group had documented influenza vaccination in the EMR, although some

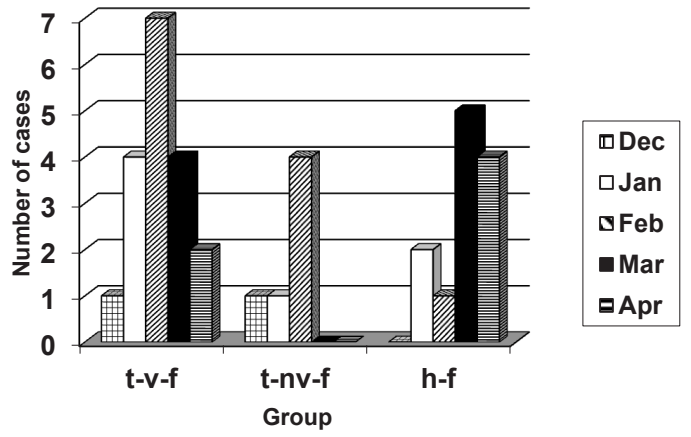

Figure 1: Month during which influenza occurred by group T-v-f=Transplant recipients who developed influenza despite influenza vaccination T-nv$\mathrm{f}=$ Transplant recipients who developed influenza in the absence of influenza vaccination $\mathrm{H}-\mathrm{f}=$ Healthy people who developed influenza.

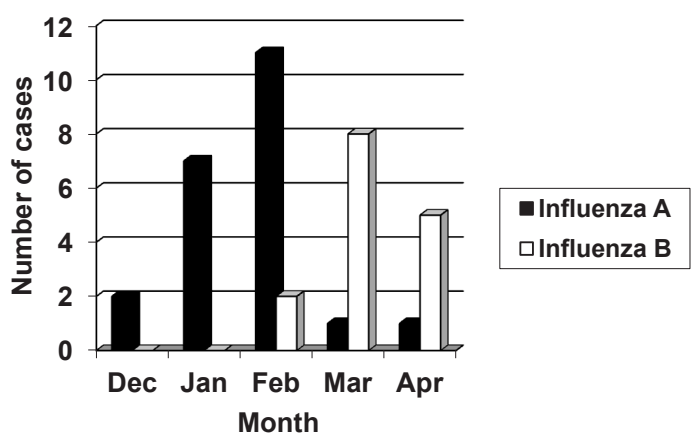

Figure 2: Epidemic curve of influenza A and B. may have received it elsewhere. All cases of influenza were communityacquired except one patient who had nosocomial influenza $\mathrm{A}$ in the txvac-flu group while awaiting heart transplant in the hospital (Table 1).

\section{Clinical characteristics}

Fever and cough were the most common presenting symptoms. There were no significant differences between the tx-vac-flu, tx-no vacflu, and healthy-flu groups in the incidence of fever, cough, headache, rhinorrhea, sore throat, malaise, shortness of breath, other symptoms, or the presence of infiltrate on chest plain radiography. Seven patients (5 lung, 1 heart \& 1 kidney pancreas recipients) in the tx-vac-flu group had pulmonary infiltrates. The incidence of symptoms of upper respiratory tract infection in contacts of patients in these groups was also not significantly different (Table 2 ).

\section{Virological characteristics}

One lung transplant recipient in the tx-vac-flu group had 1 episode of influenza A, and 1 episode of influenza B two months later, so was counted twice. One HSCT recipient in the tx-no vac-flu group had influenza A and B simultaneously. Influenza A was significantly more common in patients in the tx-vac-flu group than those in the healthyflu group (P 0.04), and the opposite was true for influenza B. Cases of influenza $\mathrm{B}$ occurred later in the season; mirroring epidemiology in the community (Figures 1 and 2). There were no significant differences in influenza PCR cycle threshold between the tx-vac-flu, tx-no vac-flu, and healthy-flu groups (Table 3 and Figure 3).

\section{Risk factors for influenza}

Proportions of patients who were transplanted within the preceding 6 months (as opposed to $>6$ months) were not significantly different among the tx-vac-flu, tx-no vac-flu, and tx-vac-no flu groups. Forced expiratory volume in 1 second (FEV1) was measured before the episode of influenza in 24 of 37 (65\%) transplant recipients (11 lung, 8 HSCT,

\begin{tabular}{|c|c|c|c|c|}
\hline & \multicolumn{4}{|c|}{ Group } \\
\hline & $\begin{array}{c}\text { Tx-vac-flu } \\
(n=18)\end{array}$ & $\begin{array}{l}\text { Tx-no vac-flu } \\
(n=6)\end{array}$ & $\begin{array}{l}\text { Healthy-flu } \\
(n=12)\end{array}$ & $\begin{array}{c}\text { Tx-vac-no flu } \\
(n=13)\end{array}$ \\
\hline Fever (n [\%]) & $14[78 \%]$ & $6[100 \%]$ & $11[92 \%]$ & \multirow{10}{*}{$\mathrm{N} / \mathrm{A}$} \\
\hline Cough (n [\%]) & 18 [100\%] & $4[67 \%]$ & $12[100 \%]$ & \\
\hline Headache (n [\%]) & $5[28 \%]$ & $3[50 \%]$ & $5[42 \%]$ & \\
\hline Rhinorrhea (n [\%]) & $10[56 \%]$ & $4[67 \%]$ & 7 [58\%] & \\
\hline Sore throat (n [\%]) & 7 [39\%] & $4[67 \%]$ & 3 [25\%] & \\
\hline Malaise (n [\%]) & $10[56 \%]$ & $6[100 \%]$ & 8 [67\%] & \\
\hline Shortness of breath (n [\%]) & $10[56 \%]$ & 2 [33\%] & 5 [42\%] & \\
\hline Other symptoms (n [\%]) & $13[72 \%]^{*}$ & $4[67 \%]^{\dagger}$ & $4[33 \%]^{\#}$ & \\
\hline Symptoms of URI in contacts ( $\mathrm{n}[\%]$ ) & 7 [39\%] & $3[50 \%]$ & $2[17 \%]^{* *}$ & \\
\hline${ }^{\dagger \dagger}$ Infiltrate on CXR (n [\%]) & 7 [39\%] & $0[0 \%]$ & $0[0 \%]$ & \\
\hline
\end{tabular}

Tx-vac-flu=Transplant recipients who developed influenza despite influenza vaccination

Tx-no vac-flu=Transplant recipients who developed influenza in the absence of influenza vaccination Healthy-flu=Healthy people who developed influenza

Tx-vac-no flu=Transplant recipients who received influenza vaccination and did not develop influenza $\mathrm{N} / \mathrm{A}=$ not applicable. URI=upper respiratory tract infection. CXR=Chest Plain Radiography.

* Other symptoms in the tx-vac-flu group were diarrhea (4), wheezing (2), rash (1), chills (1), chest pain (1), anorexia (1), hoarseness (1), ear ache (1), and dysuria (1).

tOther symptoms in the tx-no vac-flu group were anorexia (1), nausea (1), vomiting (1), and diarrhea (1)

\#other symptoms in the healthy-flu group were anorexia (1), wheezing (1), ear ache (1), and sweats (1).

*Data available from 3 patients.

${ }^{+\dagger}$ All patients in the tx-vac-flu group, 5 patients in the tx-no vac-flu group, and 5 patients in the healthy-flu group had CXR done

$\mathrm{P}=$ not significant for all comparisons to the tx-vac-flu group in this table.

Table 2: Clinical characteristics. 
2 liver, 2 heart, and 1 kidney + liver), but in none of the previously healthy patients. FEV1 was significantly shorter in patients in the txvac-flu group than those in the tx-no vac-flu group $(\mathrm{P}=0.014)$, but not the tx-vac-no flu group $(\mathrm{P}=0.064)$. There was no significant difference between the tx-vac-flu, tx-no vac-flu, and tx-vac-no flu groups when comparing the proportion of patients with FEV1<2L. Immunoglobulin $\mathrm{G}$ (IgG) levels were measured in 35 of 37 (94\%) transplant recipients, but in none of the previously healthy patients. IgG levels were not significantly different in the tx-vac-flu, tx-no vac-flu, and tx-vac-no groups. There was no significant difference between the tx-vac-fly, txno vac-flu, and tx-vac-no flu groups when comparing the proportion of patients with $\operatorname{IgG}<600 \mathrm{mg} / \mathrm{dL}$. Immune function assays (ATP levels) were measured in 26 of 37 (70\%) transplant recipients, but in none of the previously healthy patients. ATP levels were not significantly different between the tx-vac-no flu, tx-no vac-flu, and tx-vac-no flu groups. There was no significant difference between the tx-vac-no flu, tx-no vac-flu, and tx-vac-no flu groups when comparing the proportion of patients with ATP level $<200 \mathrm{ng} / \mathrm{mL}$. Proportion of patients who had rejection or graft-versus-host disease (GVHD) in the 30 days preceding the diagnosis of influenza was not significantly different between the tx-vac-flu and tx-no vac-flu groups. Patients in the tx-vac-flu group had concomitant infections significantly more frequently than the healthyflu group $(\mathrm{P}=0.043)$, but not the tx-no vac-flu group $(\mathrm{P}=0.050)$. Patients in the tx-vac-no flu group had other infections significantly more frequently than patients in the tx-vac-flu group $(\mathrm{P}=0.0009)$ (Table 4$)$.

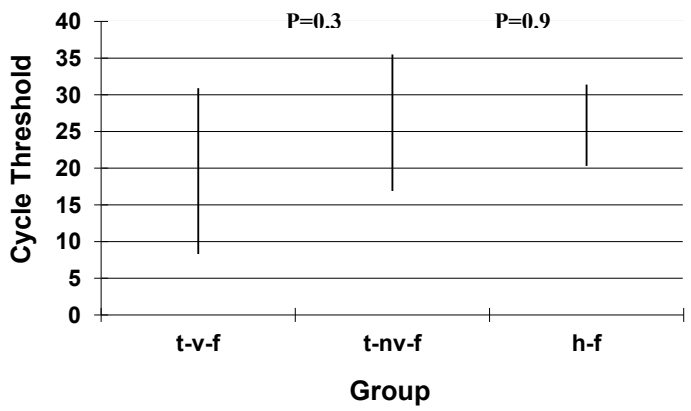

Figure 3: Range of influenza PCR cycle threshold by group T-v-f=Transplant recipients who developed influenza despite influenza vaccination T-nv$\mathrm{f}=$ Transplant recipients who developed influenza in the absence of influenza vaccination $\mathrm{H}-\mathrm{f}=\mathrm{Healthy}$ people who developed influenza.

\section{Treatment}

None of the patients were on prophylactic anti-influenza drugs before the onset of symptoms of influenza. Oseltamivir was the only drug used for treatment in all patients. Patients in the tx-vac-flu group received treatment with oseltamivir significantly more frequently than the healthy-flu group $(\mathrm{P}=0.0006)$, but not the tx-no vac-flu group $(\mathrm{P}=0.75)$. Duration of treatment with oseltamivir was not significantly different in the tx-vac-flu, tx-no vac-flu, and healthy-flu groups. Dose of oseltamivir for all patients was $75 \mathrm{mg}$ orally twice daily; except in 1 patient in the tx-flu-vac group, whose dose was $150 \mathrm{mg}$ orally twice daily. One patient in the tx-vac-flu group and 8 patients in the healthyflu group were not treated with oseltamivir or other drugs active against influenza (Table 5).

\section{Outcomes}

Patients in the tx-vac-flu group developed pneumonia following the diagnosis of influenza significantly more frequently than patients in the healthy-flu group $(\mathrm{P}=0.031)$, but not the tx-no vac-flu group $(\mathrm{P}$ $=0.137)$. One of the 6 patients with pneumonia in the tx-vac-flu group had aspergillus pneumonia, and one had pseudomonas pneumonia. Patients in the tx-vac-flu group were hospitalized more frequently than patients in the healthy-flu group $(\mathrm{P}=0.00007)$, but not the tx-no vacflu group $(\mathrm{P}=0.963)$. Length of hospitalization was not significantly different between the tx-vac-flu and the tx-no vac-flu groups. One of 18 patients $(6 \%)$ in the tx-vac-flu group and none in the tx-no vac-flu and healthy-flu groups required admission to an intensive care unit and mechanical ventilation following influenza. No patients died as a result of influenza. FEV1 was measured at a median interval of 17 months after the episode of influenza in 18 of $24(75 \%)$ transplant recipients who had FEV1 measured before the episode of influenza (11 lung, 6 HSCT, and 1 liver), but in none of the previously healthy patients. There were no significant differences in the degree of change in FEV1 between the tx-vac-flu, tx-no vac-flu, and tx-vac-no flu groups. Two patients in the tx-vac-flu group had mild acute rejection within 2 months after the diagnosis of influenza, and one had grade three skins GVHD. None of the patients in the tx-no vac-flu group had rejection or GHVD in that time frame (Table 6).

\section{Discussion}

The current study showed that the presenting symptoms of influenza in transplant recipients who developed influenza despite receiving influenza vaccination might not be different than in transplant recipients who did not receive influenza vaccination, or in

\begin{tabular}{|c|c|c|c|c|c|}
\hline & & \multicolumn{4}{|c|}{ Group } \\
\hline & & $\begin{array}{c}\text { Tx-vac-flu } \\
(n=18)\end{array}$ & $\begin{array}{c}\text { Tx-no vac-flu } \\
(n=6)\end{array}$ & $\begin{array}{l}\text { Healthy-flu } \\
(n=12)\end{array}$ & $\begin{array}{c}\text { Tx-vac-no flu } \\
(n=13)\end{array}$ \\
\hline \multirow{2}{*}{ Type of influenza } & $A$ & 13 & 5 & $4^{*}$ & \multirow{6}{*}{$\mathrm{N} / \mathrm{A}$} \\
\hline & $\mathrm{B}$ & 5 & 2 & 8 & \\
\hline \multirow{4}{*}{ Influenza PCR cycle threshold } & Mean & 23.3 & 26.6 & 24.5 & \\
\hline & Range & $8.3-30.9$ & $16.9-35.5$ & $20.3-31.4$ & \\
\hline & Median & 25.3 & $26.7^{\dagger}$ & $24.3^{\#}$ & \\
\hline & Interquartile Range & $20.6-27.5$ & $23.5-29.9$ & $20.8-26.2$ & \\
\hline
\end{tabular}

Tx-vac-flu=Transplant recipients who developed influenza despite influenza vaccination

Tx-no vac-flu=Transplant recipients who developed influenza in the absence of influenza vaccination

Healthy-flu=Healthy people who developed influenza

Tx-vac-no flu=Transplant recipients who received influenza vaccination and did not develop influenza

$\mathrm{N} / \mathrm{A}=$ not applicable.

${ }^{*} \mathrm{P}=0.04,{ }^{+} \mathrm{P}=0.3,{ }^{\#} \mathrm{P}=0.9$; compared to the tx-vac-flu group.

Table 3: Virological characteristics. 
Citation: Mossad SB, Yen-Lieberman B, Shrestha NK, Mossad DM, Mawhorter SD, et al. (2013) Characteristics of Transplant Recipients Who Developed Influenza in 2007-08 Despite Influenza Vaccination. J Vaccines Vaccin 4: 177. doi:10.4172/2157-7560.1000177

Page 5 of 8

\begin{tabular}{|c|c|c|c|c|}
\hline & \multicolumn{4}{|c|}{ Group } \\
\hline & $\begin{array}{l}\text { Tx-vac-flu } \\
(n=18)\end{array}$ & $\begin{array}{l}\text { Tx-no vac-flu } \\
\quad(n=6)\end{array}$ & $\begin{array}{l}\text { Healthy-flu } \\
(n=12)\end{array}$ & $\begin{array}{l}\text { Tx-vac-no flu } \\
\quad(n=13)\end{array}$ \\
\hline Transplant within the preceding 6 months (n [\%]) & $2[11 \%]$ & $3[50 \%]$ & \multirow{8}{*}{$\mathrm{N} / \mathrm{A}$} & $5[38 \%]$ \\
\hline${ }^{*} \mathrm{FEV} 1(\mathrm{~L})$ \{median [IQR]\} & $\begin{array}{c}1.59 \\
{[0.99-2.51]}\end{array}$ & $\begin{array}{c}3.61^{\dagger} \\
{[3-4.61]}\end{array}$ & & $\begin{array}{c}2.79 \\
{[2.14-3.39]}\end{array}$ \\
\hline${ }^{*} \mathrm{FEV} 1<2 \mathrm{~L}(\mathrm{n}[\%]$ & $5[50 \%]$ & $1[20 \%]$ & & $2[22 \%]$ \\
\hline${ }^{\#} \mathrm{IgG}(\mathrm{mg} / \mathrm{dL})\{$ median $[\mathrm{IQR}]\}$ & $\begin{array}{c}761 \\
{[484-947]}\end{array}$ & $\begin{array}{c}580 \\
{[527-654]}\end{array}$ & & $\begin{array}{c}641 \\
{[586-876]}\end{array}$ \\
\hline${ }^{\#} \operatorname{lgG}<600$ mg/dL (n [\%]) & $6[33 \%]$ & $3[50 \%]$ & & $6[50 \%]$ \\
\hline${ }^{*}$ ATP $(\mathrm{ng} / \mathrm{mL})$ immune function assay \{median [IQR]\} & $\begin{array}{c}212 \\
{[154-384]}\end{array}$ & $\begin{array}{c}199 \\
{[140-258]}\end{array}$ & & $\begin{array}{c}425 \\
{[268-446]}\end{array}$ \\
\hline${ }^{*}$ ATP immune function assay < $200 \mathrm{ng} / \mathrm{mL}$ (n [\%]) & $7[47 \%]$ & $1[50 \%]$ & & $1[11 \%]$ \\
\hline $\begin{array}{c}\text { Rejection or GVHD in the } 30 \text { days preceding } \\
\text { diagnosis of influenza ( } \mathrm{n}[\%])\end{array}$ & $1[6 \%]$ & $1[17 \%]$ & & $\mathrm{N} / \mathrm{A}$ \\
\hline Concomitant infections (n [\%]) & †+8 [44] & $0[0 \%]$ & \#1 [8\%] & $13[100 \%]^{\Delta}$ \\
\hline
\end{tabular}

Tx-vac-flu=Transplant recipients who developed influenza despite influenza vaccination

Tx-no vac-flu=Transplant recipients who developed influenza in the absence of influenza vaccination

Healthy-flu=Healthy people who developed influenza

Tx-vac-no flu=Transplant recipients who received influenza vaccination and did not develop influenza

$\mathrm{N} / \mathrm{A}=$ not applicable. FEV1=Forced expiratory volume in 1 second. IQR=interquartile range. IgG=Immunoglobulin G level. ATP=Adenosine triphosphate. GVHD=graftversus-host disease

*10 patients in the tx-vac-flu group, 5 in the tx-no vac-flu group, none in the healthy-flu group, and 9 in the tx-vac-no flu group had FEV1 measured.

${ }^{\dagger} \mathrm{P}=0.014$ compared to the tx-vac-flu group

\#17 patients in the tx-vac-flu group, 5 in the tx-no vac-flu group, none in the healthy-flu group, and 13 in the tx-vac-no flu group had IgG level measured.

Normal range for lgG level=717 - $1411 \mathrm{mg} / \mathrm{dL}$.

*15 patients in the tx-vac-flu group, 2 in the tx-no vac-flu group, none in the healthy-flu group, and 9 in the tx-vac-no flu group had ATP level measured.

Normal range for ATP level=225 $-525 \mathrm{ng} / \mathrm{mL}$

${ }^{+\dagger}$ Concomitant infections in the tx-vac-flu group were cytomegalovirus viremia (CMV) (2), Epstein-Barr virus (EBV) related posttransplant lymphoproliferative disease (2), aspergillus fumigatus pneumonia (1), methicillin-susceptible staphylococcus aureus pneumonia (1), rotavirus enteritis (1), respiratory syncytial virus upper respiratory tract infection (1), urinary tract infection (UTI) with proteus vulgaris and klebsiella pneumoniae (1), skin infection with mycobacterium cheloniae-abscessus (1),

pseudomonas aeruginosa pneumonia (1), and EBV viremia (1).

\#1 patient in the healthy-flu group had concomitant sinusitis

$\mathrm{P}=0.043$ compared to the tx-vac-flu group

Patients in the tx-vac-no flu group did not have influenza, but had CMV viremia (5), peritonitis (2), UTI (2), bacteremia (2), aspergillosis (1), sinusitis (1), EBV viremia (1), cryptococcosis (1), bacterial pneumonia (1), hepatic candidiasis (1), and clostridium-difficile-associated diarrhea (1).

${ }^{\Delta} \mathrm{P}=0.0009$ compared to the tx-vac-flu group

$\mathrm{P}=$ not significant for all other comparisons to the tx-vac-flu group in this table.

Table 4: Risk factors for influenza

\begin{tabular}{|c|c|c|c|c|}
\hline & \multicolumn{4}{|c|}{ Group } \\
\hline & $\begin{array}{c}\text { Tx-vac-flu } \\
(n=18)\end{array}$ & $\begin{array}{c}\text { Tx-no vac-flu } \\
(n=6)\end{array}$ & $\begin{array}{l}\text { Healthy-flu } \\
(n=12)\end{array}$ & $\begin{array}{c}\text { Tx-vac-no flu } \\
(n=13)\end{array}$ \\
\hline Number of patients treated with oseltamivir (\%) & $17(94 \%)$ & $6(100 \%)$ & $4(33 \%)^{*}$ & \multirow{2}{*}{$\mathrm{N} / \mathrm{A}$} \\
\hline Duration of treatment with oseltamivir (days) \{median [IQR]\} & $5[5-7]$ & $7.5[5-10]$ & $5[5]$ & \\
\hline
\end{tabular}

Tx-vac-flu=Transplant recipients who developed influenza despite influenza vaccination

Tx-no vac-flu=Transplant recipients who developed influenza in the absence of influenza vaccination

Healthy-flu=Healthy people who developed influenza

Tx-vac-no flu=Transplant recipients who received influenza vaccination and did not develop influenza

$\mathrm{N} / \mathrm{A}=$ not applicable. IQR=interquartile range

${ }^{*} \mathrm{P}=0.0006$ compared to the tx-vac-flu group

$\mathrm{P}=$ not significant for all other comparisons to the tx-vac-flu group in this table.

Table 5: Treatment.

previously healthy people. Older age and shorter FEV1 were associated with influenza vaccine failure in transplant recipients. These are logical findings, since older patients, and those with underlying lung disease are at higher risk of influenza-related complications. Although influenza occurred after a longer interval from transplantation in transplant recipients who developed influenza despite receiving influenza vaccination than transplant recipients who developed influenza without antecedent influenza vaccination, there was slightly higher incidence of pneumonia in transplant recipients who developed influenza despite receiving influenza vaccination. Factors not assessed such interval from onset of symptoms, degree of lymphopenia and type of immunosuppression may account for this difference. Surprisingly, the degree of immunosuppression using $\operatorname{IgG}$ and ATP levels as surrogate markers was not a risk factor for influenza vaccine failure. As expected, transplant patients who developed influenza despite receiving influenza vaccination were treated with oseltamivir more frequently 


\begin{tabular}{|c|c|c|c|c|}
\hline & \multicolumn{4}{|c|}{ Group } \\
\hline & $\begin{array}{c}\text { Tx-vac-flu } \\
(n=8)\end{array}$ & $\begin{array}{l}\text { Tx-no vac-flu } \\
(n=6)\end{array}$ & $\begin{array}{l}\text { Healthy-flu } \\
(n=12)\end{array}$ & $\begin{array}{c}\text { Tx-vac-no flu } \\
(n=13)\end{array}$ \\
\hline Pneumonia (n [\%]) & $6[33 \%]$ & $0[0 \%]$ & $0[0 \%]^{*}$ & \\
\hline Hospitalization (n [\%]) & $15[83 \%]$ & $5[83 \%]$ & $1[8 \%]^{\dagger}$ & \\
\hline $\begin{array}{l}\text { Length of hospitalization in days } \\
\text { Median } \\
\text { Mean } \\
\text { Range }\end{array}$ & $\begin{array}{c}7.2 \\
4 \\
2-30\end{array}$ & $\begin{array}{c}3.4^{\#} \\
3 \\
3-4\end{array}$ & N/A & N/A \\
\hline Admission to an ICU (n [\%]) & $1[6 \%]$ & $0[0 \%]$ & $0[0 \%]$ & \\
\hline Need for mechanical ventilation (n [\%]) & $1[6 \%]$ & $0[0 \%]$ & $0[0 \%]$ & \\
\hline "FEV1 (L) change (median ) at a later date & +0.035 & -0.73 & $\mathrm{~N} / \mathrm{A}$ & -0.22 \\
\hline
\end{tabular}

Tx-vac-flu=Transplant recipients who developed influenza despite influenza vaccination

Tx-no vac-flu=Transplant recipients who developed influenza in the absence of influenza vaccination

Healthy-flu=Healthy people who developed influenza

Tx-vac-no flu=Transplant recipients who received influenza vaccination and did not develop influenza

$\mathrm{N} / \mathrm{A}=$ not applicable. ICU=intensive care unit. FEV1=Forced expiratory volume in 1 second.

** 8 patients in the tx-vac-flu group, 4 in the tx-no vac-flu group, none in the healthy-flu group, and 6 in the tx-vac-no flu group had FEV1 measured after a median of 17 months.

" $\mathrm{P}=0.031,{ }^{\dagger} \mathrm{P}=0.00007,{ }^{*} \mathrm{P}=0.19$, compared to the tx-vac-flu group

$\mathrm{P}=$ not significant for all other comparisons to the tx-vac-flu group in this table.

Table 6: Outcomes.

than previously healthy people, but the duration of therapy was not longer. Other expected findings included that transplant patients who developed influenza despite receiving influenza vaccination were hospitalized, developed pneumonia, and had concomitant infections more frequently than previously healthy patients.

Limitations of this study include its retrospective nature, with the associated gaps in data collected; particularly in previously healthy patients, and uncontrolled interventions. For example, some, but not all transplant recipients, and none of the healthy patients had FEV1 measured before and after transplantation. The type and intensity of exposure to influenza virus in each group, and whether they utilized personal protective behaviors are not known. Although the comparator groups were randomly identified, selection bias cannot be excluded. The groups were not matched, so other risk factors may have impacted the outcomes. The number of patients was small in some comparisons, so other significant differences may have been detected with larger numbers. Assays to assess influenza-specific immunity were not done, thus influenza vaccine failure cannot be claimed as causative for any of the outcomes. Data on influenza immunizations prior to the 2007-08 seasons were not available, so "archiving immunity" cannot be assessed.

Although clinical findings may identify patients with ILI, they cannot confirm or exclude the diagnosis of influenza [25]; particularly in hospitalized patients [26]. Accurate and rapid diagnosis of influenza is not only important for timely prescribing of specific antiviral therapy, but also to prevent nosocomial [27] and household [28] transmission, and reducing unnecessary antibacterial therapy [29].

Effectiveness of influenza vaccine depends on the degree of antigenic match to circulating influenza viruses [30]. Historically, a serum hemagglutination-inhibiting antibody (HIA) titer $>1: 40$ in response to vaccination in healthy individuals have been considered protective against infection [31]. However, this HIA titer may not be protective in elderly or immunocompromised individuals. A higher dose of influenza vaccine has been shown to be more immunogenic in elderly persons, but is associated with a significant increase in injection site reactions [32]. Increasing the influenza vaccine dosage has also been shown to induce increasing levels of cross-reacting antibodies to subsequent, antigenically different influenza variants, including some appearing $>10$ years after vaccination [33]. In adults $>60$ years of age, intradermal influenza vaccination was shown to be more immunogenic than intramuscular administration, but whether that results in enhanced protection in this vulnerable population is not known [34].

Influenza vaccination of transplant candidates is vital to their protection in the early post transplant period [10]. Certainly, better measures to prevent and treat influenza in transplant recipients are needed. While some studies have shown up to $80 \%$ vaccine efficacy [9] in HSCT recipients, others have shown poor serological responses within the first 2 years after transplant [35]. A booster dose of influenza vaccine did not enhance seroprotection or seroresponse in renal transplant recipients [5]. Similarly, a two-dose regimen of influenza vaccine in HSCT recipients only marginally enhanced immunological response [36], as did granulocyte-macrophage colony-stimulating factor; administered as an immunomodulating agent with influenza vaccine [37]. While HIA titer has been traditionally used to measure protective response to influenza vaccination [31] assessment of cell-mediated immune response [38] should also be considered in transplant recipients. Intradermal administration of influenza vaccine using a microinjection system [39] may be specifically appealing in thrombocytopenic HSCT recipients, and coagulopathic liver transplant recipients. Although an intradermal boosting strategy for influenza vaccination using $3 \mu \mathrm{g}$ of hemagglutinin antigen per influenza strain in lung transplant recipients did not significantly improve vaccine immunogenicity [40] intradermal influenza vaccination using $15 \mu \mathrm{g}$ of hemagglutinin antigen per influenza strain was immunogenic in renal transplant recipients who had previously not responded to subcutaneous influenza vaccination [41]. An adjuvanted 2009 pandemic influenza A/H1N1 vaccine was less immunogenic in SOT recipients than healthy controls [42]. Influenza vaccination of health care providers [43] and household contacts [44] of transplant candidate and recipients is imperative to create a "circle of protection" around this vulnerable population. A recent study showed that influenza vaccination of children and adolescents with inactivated influenza vaccine significantly protected even unimmunized residents of rural communities [45].

Nonpharmacological interventions, including facemasks and 
Citation: Mossad SB, Yen-Lieberman B, Shrestha NK, Mossad DM, Mawhorter SD, et al. (2013) Characteristics of Transplant Recipients Who Developed Influenza in 2007-08 Despite Influenza Vaccination. J Vaccines Vaccin 4: 177. doi:10.4172/2157-7560.1000177

hand hygiene [46] play an important role in preventing influenza transmission.

While some studies have shown the effectiveness of oseltamivir for treatment of influenza in transplant recipients [14,15], and some support its use for seasonal prophylaxis [16] rational use of antiviral agents is necessary, since monotherapy may predispose to mutational pressure and selection of antiviral-resistant strains [47]. New antiviral agents for influenza treatment for the general population and specifically for transplant recipients are awaited [48]. The 2009 influenza A/ $\mathrm{H} 1 \mathrm{~N} 1$ pandemic has increased awareness and improved guidance for management of influenza in transplant recipients [49].

\section{References}

1. Lopez-Medrano F, Aguado JM, Lizasoain M, Folgueira D, Juan RS, et al. (2007) Clinical implications of respiratory virus infections in solid organ transplant recipients: a prospective study. Transplantation 84: 851-856.

2. Chemaly RF, Ghosh S, Bodey GP, Rohatgi N, Safdar A, et al. (2006) Respiratory viral infections in adults with hematologic malignancies and human stem cell transplantation recipients: $A$ retrospective study at a major cancer center. Medicine (Baltimore) 85: 278-287.

3. Mazzone PJ, Mossad SB, Mawhorter SD, Mehta AC, Schilz RJ, et al. (2001) The humoral immune response to influenza vaccination in lung transplant patients. Eur Respir J 18: 971-976.

4. Mazzone PJ, Mossad SB, Mawhorter SD, Mehta AC, Mauer JR (2004) Cell-mediated immune response to influenza vaccination in lung transplan recipients. J Heart Lung Transplant 23: 1175-1181.

5. Scharpe J, Evenepoel P, Maes B, Bammens B, Claes K, et al. (2008) Influenza vaccination is efficacious and safe in renal transplant recipients. Am J Transplant 8: 332-337.

6. Madan RP, Tan M, Fernandez-Sesma A, Moran TM, Emre S, et al. (2008) A prospective, comparative study of the immune response to inactivated influenza vaccine in pediatric liver transplant recipients and their healthy siblings. Clin Infect Dis 46: 712-718.

7. Dopp JM, Wiegert NA, Moran JJ, Francois ML, Radford KL, et al. (2009) Effect of annual influenza immunization on antibody response in lung transplant patients. Prog Transplant 19: 153-159.

8. Magnani G, Falchetti E, Pollini G, Reggiani LB, Grigioni F, et al. (2005) Safety and efficacy of two types of influenza vaccination in heart transplant recipients: A prospective randomized controlled study. J Heart Lung Transplant 24: 588592

9. Machado CM, Cardoso MR, da Rocha IF, Boas LS, Dulley FL, et al. (2005) The benefit of influenza vaccination after bone marrow transplantation. Bone Marrow Transplant 36: 897-900.

10. Kumar D, Blumberg EA, Danziger-Isakov L, Kotton CN, Halasa NB, et al. (2011) Influenza vaccination in the organ transplant recipient: Review and summary recommendations? Am J Transplant 11: 2020-2030.

11. Ljungman $\mathrm{P}$, Cordonnier $\mathrm{C}$, Einsele $\mathrm{H}$, Englund J, Machado CM, et al. (2009) Vaccination of hematopoietic cell transplant recipients. Bone Marrow Transplant 44: $521-526$

12. Willcocks LC, Chaudhry AN, Smith JC, Ojha S, Doffinger R, et al. (2007) The effect of sirolimus therapy on vaccine responses in transplant recipients. Am J Transplant 7: 2006-2011.

13. Vu D, Peck AJ, Nichols WG, Varley C, Englund JA, et al. (2007) Safety and tolerability of oseltamivir prophylaxis in hematopoietic stem cell transplant recipients: A retrospective case-control study. Clin Infect Dis 45: 187-193.

14. Khanna N, Steffen I, Studt JD, Schreiber A, Lehmann T, et al. (2009) Outcome of influenza infections in outpatients after allogeneic hematopoietic stem cell transplantation. Transpl Infect Dis 11: 100-105.

15. Ison MG, Sharma A, Shepard JA, Wain JC, Ginns LC (2008) Outcome of influenza infection managed with oseltamivir in lung transplant recipients. J Heart Lung Transplant 27: 282-288.

16. Ison MG, Szakaly P, Shapira MY, Krivan G, Nist A, et al. (2012) Efficacy and safety of oral oseltamivir for influenza prophylaxis in transplant recipients. Antivir Ther 17: 955-964
17. Hoffmann-La Roche (2013) A study of tamiflu (oseltamivir) for treatment of influenza in immunocompromised patients.

18. Medeiros R, Rameix-Welti MA, Lorin V, Ribaud P, Manuguerra JC, et al. (2007) Failure of zanamivir therapy for pneumonia in a bone-marrow transplant recipient infected by a zanamivir-sensitive influenza A (H1N1) virus. Antivir Ther 12: $571-576$.

19. Watcharananan SP, Suwatanapongched T, Wacharawanichkul P, Chantratitaya W, Mavichak V, et al. (2010) Influenza A/H1N1 2009 pneumonia in kidney transplant recipients: Characteristics and outcomes following high-dose oseltamivir exposure. Transpl Infect Dis 12: 127-131.

20. Kumar D, Michaels MG, Morris MI, Green M, Avery RK, et al. (2010) Outcomes from pandemic influenza A H1N1 infection in recipients of solid-organ transplants: A multicentre cohort study. Lancet Infect Dis 10: 521-526.

21. Memoli MJ, Hrabal RJ, Hassantoufighi A, Eichelberger MC, Taubenberger JK (2010) Rapid selection of oseltamivir- and peramivir-resistant pandemic H1N1 virus during therapy in 2 immunocompromised hosts. Clin Infect Dis 50: 12521255.

22. Kumar D, Humar A (2006) Pandemic influenza and its implications for transplantation. Am J Transplant 6: 1512-1517.

23. Centers for Disease Control and Prevention (CDC) (2008) Interim withinseason estimate of the effectiveness of trivalent inactivated influenza vaccine-marshfield, wisconsin, 2007-08 influenza season. MMWR Morb Mortal Wkly Rep 57: 393-398.

24. LeGoff J, Kara R, Moulin F, Si-Mohamed A, Krivine A, et al. (2008) Evaluation of the one-step multiplex real-time reverse transcription-PCR ProFlu-1 assay for detection of influenza A and influenza B viruses and respiratory syncytial viruses in children. J Clin Microbiol 46: 789-791.

25. Call SAMSPH, Vollenweider MA, Hornung CA, Simel DLMHS, McKinney WP (2005) Does this patient have influenza? JAMA 293: 987-997.

26. van den Dool C, Hak E, Wallinga J, van Loon AM, Lammers JW, et al. (2008) Symptoms of influenza virus infection in hospitalized patients. Infect Contro Hosp Epidemiol 29: 314-319.

27. Gooskens J, Jonges M, Claas ECJ, Meijer A, van den Broek PJ, et al. (2009) Morbidity and mortality associated with nosocomial transmission of oseltamivirresistant influenza A (H1N1) virus. JAMA 301: 1042-1046.

28. France A, Jackson M, Schrag S, Lynch M, Zimmerman C, et al. (2010) Household transmission of 2009 influenza A (H1N1) virus after a School Based outbreak in New York city, April-May 2009. J Infect Dis 201: 984-992.

29. Falsey AR, Murata Y, Walsh EE (2007) Impact of rapid diagnosis on management of adults hospitalized with influenza. Arch Intern Med 167: 354 360.

30. Belongia E, Kieke B, Donahue J, Greenlee RT, Balish A, et al. (2009) Effectiveness of inactivated influenza vaccines varied substantially with antigenic match from the 2004-2005 season to the 2006-2007 season. J Infect Dis 199: 159-167.

31. Hobson D, Curry RL, Beare AS, Ward-Gardner A (1972) The role of serum haemagglutination-inhibiting antibody in protection against challenge infection with influenza A2 and B viruses. J Hyg (Lond) 70: 767-777.

32. Keitel WA, Atmar RL, Cate TR, Petersen NJ, Greenberg SB, et al. (2006) Safety of high doses of influenza vaccine and effect on antibody responses in elderly persons. Arch Intern Med 166: 1121-1127.

33. Keitel WA, Atmar RL, Nino D, Cate TR, Couch RB (2008) Increasing doses of an inactivated influenza $\mathrm{A} / \mathrm{H} 1 \mathrm{~N} 1$ vaccine induce increasing levels of crossreacting antibody to subsequent, antigenically different, variants. J Infect Dis 198: 1016-1018.

34. Holland D, Booy R, Looze FD, Eizenberg P, McDonald J, et al. (2008) Intradermal influenza vaccine administered using a new microinjection system produces superior immunogenicity in elderly adults: A randomized controlled trial. J Infect Dis 198: 650-658.

35. Gandhi MK, Egner W, Sizer L, Inman I, Zambon M, et al. (2001) Antibody responses to vaccinations given within the first two years after transplant is similar between autologous peripheral blood stem cell and bone marrow transplant recipients. Bone Marrow Transplant 28: 775-781.

36. Engelhard D, Nagler A, Hardan I, Morag A, Aker M, et al. (1993) Antibody 
Citation: Mossad SB, Yen-Lieberman B, Shrestha NK, Mossad DM, Mawhorter SD, et al. (2013) Characteristics of Transplant Recipients Who Developed Influenza in 2007-08 Despite Influenza Vaccination. J Vaccines Vaccin 4: 177. doi:10.4172/2157-7560.1000177

response to a two-dose regimen of influenza vaccine in allogeneic $\mathrm{T}$ celldepleted and autologous BMT recipients. Bone Marrow Transplant 11: 1-5

37. Pauksen K, Linde A, Hammarström V, Sjölin J, Carneskog J, et al. (2000) Granulocyte Macrophage Colony Stimulating factor as immunomodulating factor together with influenza vaccination in stem cell transplant patients. Clin Infect Dis 30: 342-348.

38. Avetisyan G, Aschan J, Hassan M, Ljungman P (2008) Evaluation of immune responses to seasonal influenza vaccination in healthy volunteers and in patients after stem cell transplantation. Transplantation 86: 257-263.

39. Laurent PE, Bonnet S, Alchas P, Regolini P, Mikszta JA, et al. (2007) Evaluation of the clinical performance of a new intradermal vaccine administration technique and associated delivery system. Vaccine 25: 8833-8842.

40. Manuel O, Humar A, Chen MH, Chernenko S, Singer LG, et al. (2007) Immunogenicity and safety of an intradermal boosting strategy for vaccination against influenza in lung transplant recipients. Am J Transplant 7: 2567-2572.

41. Morelon E, Noble CP, Goujon-Henry C, Daoud S, Cahen R, et al. (2010) Immunogenicity and safety of intradermal influenza vaccination in rena transplant patients who were non-responders to conventional influenza vaccination. Vaccine 28: 6885-6890.

42. Manuel O, Pascual M, Hoschler K, Giulieri S, Alves D, et al. (2011) Humora response to the influenza $\mathrm{A}$ H1N1/09 monovalent AS03-adjuvanted vaccine in immunocompromised patients. Clin Infect Dis 52: 248-256.
43. Chon WJ, Kadambi PV, Harland RC, Thistlethwaite JR, West BL, et al. (2010) Changing attitudes toward influenza vaccination in U.S. kidney transplant programs over the past decade. Clin J Am Soc Nephrol 5: 1637-1641.

44. Ferguson PE, Jordens CF, Gilroy NM (2010) Patient and family education in HSCT: Improving awareness of respiratory virus infection and influenza vaccination. A descriptive study and brief intervention. Bone Marrow Transplant 45: 656-661.

45. Loeb M, Russell ML, Moss L, Fonseca K, Fox J, et al. (2010) Effect of influenza vaccination of children on infection rates in hutterite communities: A randomized trial. JAMA 303: 943-950.

46. Cowling BJ, Chan K, Fang VJ, Cheng CK, Fung RO, et al. (2009) Facemasks and hand hygiene to prevent influenza transmission in households: A cluster randomized trial. Ann Intern Med 151: 437-446.

47. Poland GA, Jacobson RM, Ovsyannikova IG (2009) Influenza virus resistance to antiviral agents: A plea for rational use. Clin Infect Dis 48: 1254-1256.

48. Hayden $F$ (2009) Developing new antiviral agents for influenza treatment: What does the future hold? Clin Infect Dis 1: S3-13.

49. Kumar D, Morris MI, Kotton CN, Fischer SA, Michaels MG, et al. (2010) Guidance on novel influenza A/H1N1 in solid organ transplant Recipients. Am J Transplant 10: 18-25. 